\title{
Inviscid incompressible limit for the strong stratified flow of a chemically reacting gaseous mixture
}

\author{
Young-Sam Kwon \\ Department of Mathematics, Dong-A University, Busan 604-714, Korea.
}

\author{
Communicated by V. K. Le
}

\begin{abstract}
The flow of chemically reacting gaseous mixture is associated with a variety of phenomena and processes. In this paper we study the inviscid incompressible limit for the strong stratified flow of chemically reacting gaseous mixture with the ill-prepared initial data in the whole space. (C)2017 All rights reserved.
\end{abstract}

Keywords: Inviscid incompressible limit.

2010 MSC: 35L15, 35L53.

\section{Introduction}

The flow of chemically reacting gaseous mixture arises in sciences and engineering and is associated with a variety of phenomena and processes: pollutant formation, biotechnology, fuel droplets in combustion, sprays, and astrophysical plasma. Analyzing the physical regimes associated with various processes unfolds complex chemistry mechanisms and detailed transport phenomena. Many interesting problems in that context involve the behavior of solutions to the governing equations for multicomponent reactive flows as certain parameters vanish or become infinity. The objective of this work is to investigate singular limits for such complex flows based on the relative entropy in the whole space.

As a physical model of fluids, we here consider the flow of chemically reacting gaseous mixture in the whole space $\Omega=\mathbb{R}^{3}$ :

$$
\begin{aligned}
\partial_{\mathrm{t}} \rho+\operatorname{div}(\rho \mathbf{u}) & =0, \\
\partial_{\mathrm{t}}(\rho \mathbf{u})+\operatorname{div}(\rho \mathbf{u} \otimes \mathbf{u})+\frac{1}{\gamma} \nabla \rho^{\gamma} & =\mu \Delta \mathbf{u}+(\mu+v) \nabla \operatorname{div} \mathbf{u}+\rho \nabla \mathrm{G}, \\
\partial_{\mathrm{t}}(\rho Y)+\operatorname{div}(\rho Y \mathbf{u}) & =\operatorname{div}(\mathcal{F})-k \rho Y,
\end{aligned}
$$

where $\mathbf{u}$ is the vector field, $\gamma>3 / 2, \rho$ is the density, $\mathrm{Y}$ is the reactant fraction, and $\mathcal{F}=\mathrm{d} \Delta \mathrm{Y}$ denotes the diffusion flux. To begin with, we introduce the scaling limit:

$$
\mathrm{t} \mapsto \epsilon \mathrm{t}, \mathbf{u} \mapsto \varepsilon \mathbf{u}, \mu \mapsto \varepsilon \mu, v \mapsto \varepsilon v, \mathrm{~d} \mapsto \varepsilon \mathrm{d}, \mathrm{K} \mapsto \varepsilon \kappa .
$$

Email address: ykwon@dau.ac.kr (Young-Sam Kwon)

doi:10.22436/jnsa.010.05.49 
With the scaling of (1.3), the system (1.1)-(1.2) reads as follows:

$$
\begin{aligned}
\partial_{\mathrm{t}} \rho_{\varepsilon}+\operatorname{div}\left(\rho_{\varepsilon} \mathbf{u}_{\varepsilon}\right) & =0, \\
\partial_{\mathrm{t}}\left(\rho_{\varepsilon} \mathbf{u}_{\varepsilon}\right)+\operatorname{div}\left(\rho_{\varepsilon} \mathbf{u}_{\varepsilon} \otimes \mathbf{u}_{\varepsilon}\right)+\frac{1}{\varepsilon^{2} \gamma} \nabla \rho_{\varepsilon}^{\gamma} & =\mu_{\varepsilon} \Delta \mathbf{u}_{\epsilon}+\left(\mu_{\varepsilon}+v_{\varepsilon}\right) \nabla \operatorname{div} \mathbf{u}_{\epsilon}+\frac{1}{\varepsilon^{2}} \rho_{\epsilon} \nabla \mathrm{G}, \\
\partial_{\mathrm{t}}\left(\rho_{\epsilon} Y_{\varepsilon}\right)+\operatorname{div}\left(\rho_{\epsilon} Y_{\varepsilon} \mathbf{u}_{\epsilon}\right) & =d_{\varepsilon} \Delta Y_{\varepsilon}-k_{\varepsilon} \rho_{\epsilon} Y_{\varepsilon} .
\end{aligned}
$$

We also assume that

$$
\mu_{\varepsilon} \longrightarrow 0, v_{\varepsilon} \longrightarrow 0, d_{\varepsilon} \longrightarrow 0, \kappa_{\varepsilon} \longrightarrow 0,
$$

as $\varepsilon$ tends to 0 . Note that the scalings of $d_{\varepsilon}, \kappa_{\varepsilon}$ are motivated by $[3,7]$. Assume that the initial data have the following property at infinity:

$$
\rho_{\epsilon}(x) \rightarrow \bar{\rho}, \mathbf{u}_{\epsilon}(x) \rightarrow 0 \text { as }|x| \rightarrow \infty .
$$

The existence of global weak solution for the compressible flow of chemically reacting gaseous mixture (1.4)-(1.6) was proved by Donatelli and Trivisa [1, 2].

It should be pointed out that this kind of problem was first studied by Masmoudi [11] and then there are a lot of progressive works on this topic by Feireisl and Novotný [6] for the compressible Navier-StokesFourier system and by Jiang et al. [8-10] for the compressible magnetohydrodynamic flows. Recently, Feireisl et al. [5] have studied the inviscid incompressible limit of the weak solutions to the compressible Navier-Stokes equations of compressible flows with strong stratification using the relative entropy method. In this paper, we study the inviscid incompressible limit of the compressible strong startified flow of chemically reacting gaseous mixture (1.4)-(1.6) in the 3-dimensional whole space to the strong incompressible Euler equation when the number $\varepsilon$ is very small and we use the ill-prepared initial data. Our contribution of this paper is physically to derive the relative entropy which plays an important role in the proof and derive a rigorous Euler equation. This paper is a first try for the inviscid incompressible limit problems for the compressible flow of chemically reacting gaseous mixture.

Formally, the limit of the density $\tilde{\rho}$ is determined by the static problem

$$
\nabla_{x} \tilde{\rho}^{\gamma}=\tilde{\rho} \nabla_{x} G, \tilde{\rho}(x) \rightarrow \bar{\rho} \text { as }|x| \rightarrow \infty,
$$

and we will also investigate the limits

$$
\rho_{\epsilon} \rightarrow \tilde{\rho}, \sqrt{\rho_{\epsilon}} Y_{\mathcal{E}} \rightarrow \sqrt{\tilde{\rho}} Y, \sqrt{\rho_{\epsilon}} \mathbf{u}_{\epsilon} \rightarrow \sqrt{\tilde{\rho} \mathbf{v}},
$$

as $\epsilon$ tends to 0 in the suitable sense such that the given limit $(\mathbf{v}, Y)$ represents the unique strong solution of the following system on $\left[0, T_{*}\right)$ :

$$
\begin{aligned}
\partial_{\mathrm{t}} \mathbf{v}+(\mathbf{u} \cdot \nabla) \mathbf{v}+\nabla \Pi=0, & \operatorname{div}(\tilde{\rho} \mathbf{v})=0, \\
\partial_{\mathrm{t}} \mathrm{Y}+\mathbf{v} \cdot \nabla \mathrm{Y} & =0 .
\end{aligned}
$$

For the given initial $\mathbf{v}_{0}$ such that

$$
\mathbf{u}_{0} \in H^{\mathrm{k}}\left(\Omega ; \mathbb{R}^{3}\right), \mathrm{Y}_{0} \in \mathrm{H}^{\mathrm{k}}(\Omega), \operatorname{div} \mathbf{v}_{0}=0
$$

for $k>\frac{5}{2}$, the velocity $(\mathbf{v}, Y)$ has the following regularity:

$$
\begin{aligned}
& Y \in C\left(\left[0, T_{*}\right) ; H^{k}(\Omega)\right), \partial_{t} Y \in C\left(\left[0, T_{*}\right) ; H^{k-1}(\Omega)\right), \\
& \mathbf{v} \in C\left(\left[0, T_{*}\right) ; H^{k}\left(\Omega ; \mathbb{R}^{3}\right)\right), \partial_{t} \mathbf{v}, \nabla \Pi \in C\left(\left[0, T_{*}\right) ; H^{k-1}\left(\Omega ; \mathbb{R}^{3}\right)\right),
\end{aligned}
$$

on $\left[0, T_{*}\right)$, see Donatelli and Trivisa [3].

The outline of this article is as follows: In Section 2 we present the rigorous result for (1.8) and (1.9), and in Section 3 we give the proof of the main result for the compressible flow of chemically reacting gaseous mixture (1.4)-(1.6). 
Definition 1.1. We say that a quantity $\{\rho, \mathbf{u}, Y\}$ is a weak solution of the flow of a gaseous mixture (1.4)-(1.6) supplemented with the initial data $\left\{\rho_{0}, \mathbf{u}_{0}, Y_{0}\right\}$ provided that the followings hold.

- The density $\rho$ is a non-negative function, $\rho-1 \in \mathrm{L}^{\infty}\left(0, \mathrm{~T} ;\left(\mathrm{L}^{\gamma}+\mathrm{L}^{2}\right)(\Omega)\right)$, the velocity field $\mathbf{u} \in$ $\mathrm{L}^{2}\left(0, \mathrm{~T} ; \mathrm{W}^{1,2}\left(\Omega ; \mathbb{R}^{3}\right)\right), \rho|\mathbf{u}|^{2} \in \mathrm{L}^{\infty}\left(0, \mathrm{~T} ; \mathrm{L}^{1}(\Omega)\right)$, and $\mathbf{u}$ represents a renormalized solution of equation (1.4) on the $(0, T) \times \Omega$, that is, the integral identity

$$
\begin{aligned}
& \int_{\Omega}(\rho+\mathrm{b}(\rho)) \varphi(\tau, \cdot) \mathrm{d} x-\int_{\Omega}\left(\rho_{0}+\mathrm{b}\left(\rho_{0}\right)\right) \varphi(0, \cdot) \mathrm{d} x \\
& \quad=\int_{0}^{\mathrm{T}} \int_{\Omega}\left[(\rho+\mathrm{b}(\rho)) \partial_{\mathrm{t}} \varphi+(\rho+\mathrm{b}(\rho)) \mathbf{u} \cdot \nabla \varphi+\left(\mathrm{b}(\rho)-\mathrm{b}^{\prime}(\rho) \rho\right) \operatorname{div} \mathbf{u} \varphi\right] \mathrm{d} x \mathrm{dt}
\end{aligned}
$$

holds for any test function $\varphi \in \mathcal{D}([0, \mathrm{~T}) \times \Omega)$ and any $\mathrm{b}$ such that

$$
\mathrm{b} \in \mathrm{C}^{1}[0, \infty), \mathrm{b}^{\prime}(\mathrm{r})=0 \text { whenever } \mathrm{r} \geqslant \mathrm{r}_{\mathrm{b}}
$$

- The balance of momentum holds in distributional sense, namely

$$
\begin{aligned}
& \int_{\Omega} \rho \mathbf{u} \cdot \vec{\varphi}(\tau, \cdot) \mathrm{d} x-\int_{\Omega}(\rho \mathbf{u})_{0} \cdot \vec{\varphi}(0, \cdot) \mathrm{d} x \\
& =\int_{0}^{T} \int_{\Omega}\left(\rho \mathbf{u} \cdot \partial_{\mathrm{t}} \vec{\varphi}+\rho \mathbf{u} \otimes \mathbf{u}: \nabla \vec{\varphi}+\frac{1}{\gamma \epsilon^{2}} \rho^{\gamma} \operatorname{div} \vec{\varphi}\right) \mathrm{d} x \mathrm{dt} \\
& =\int_{0}^{T} \int_{\Omega} \mu_{\varepsilon} \nabla \mathbf{u}: \nabla \vec{\varphi}+\left(\mu_{\varepsilon}+v_{\varepsilon}\right) \operatorname{div} \mathbf{u} \operatorname{div} \vec{\varphi}-\frac{1}{\varepsilon^{2}} \rho \nabla \mathrm{G} \cdot \varphi \mathrm{d} x \mathrm{dt}
\end{aligned}
$$

for any test function $\vec{\varphi} \in \mathcal{D}\left([0, T) ; \mathbb{R}^{3}\right)$.

- The total energy of the system holds,

$$
\begin{gathered}
\int_{\Omega}\left(\frac{1}{2} \rho|\mathbf{u}|^{2}+\frac{1}{\gamma(\gamma-1) \varepsilon^{2}}\left(\rho^{\gamma}-\tilde{\rho}^{\gamma}-\gamma \tilde{\rho}^{\gamma-1}(\rho-\tilde{\rho})\right)\right)(\mathrm{t}, \cdot) \mathrm{d} x \\
\quad+\int_{0}^{\tau} \int_{\Omega} \mu_{\varepsilon}|\nabla \mathbf{u}|^{2}+\left(\mu_{\varepsilon}+v_{\varepsilon}\right)(\operatorname{div} \mathbf{u})^{2} \mathrm{~d} x \mathrm{dt} \leqslant \mathrm{E}_{0, \epsilon}
\end{gathered}
$$

holds for a.e. $\tau \in(0, T)$ where

$$
\mathrm{E}_{0, \epsilon}=\int_{\Omega}\left(\frac{1}{2} \rho_{0}\left|\mathbf{u}_{0}\right|^{2}+\frac{1}{\gamma(\gamma-1) \varepsilon^{2}}\left(\rho^{\gamma}-\tilde{\rho}^{\gamma}-\gamma \tilde{\rho}^{\gamma-1}\left(\rho_{0}-\tilde{\rho}\right)\right)\right) \mathrm{d} x .
$$

- The reactant mass fraction $Y$ is a bounded measurable function on $(0, T) \times \Omega$,

$$
0 \leqslant Y(t, x) \leqslant 1 \text { for a.e. } t \in(0, T), x \in \Omega,
$$

and the integral identity

$$
-\int_{0}^{T} \int_{\Omega}\left(\rho Y \partial_{t} \varphi+\rho Y u \cdot \nabla \varphi-d_{\varepsilon} \nabla Y \cdot \nabla \varphi\right) d x d t=\kappa_{\varepsilon} \int_{0}^{T} \int_{\Omega} \rho Y \varphi d x d t+\int_{\Omega} \rho_{0} Y_{0} \varphi(0, \cdot) d x,
$$

to be satisfied for any test function $\varphi \in \mathcal{D}([0, T) \times \bar{\Omega})$, together with

$$
\begin{gathered}
-\int_{0}^{T} \partial_{t} \psi \int_{\Omega} \rho \mathrm{G}(\mathbf{Y}) \mathrm{d} x \mathrm{dt}+\int_{0}^{T} \psi \int_{\Omega} \mathrm{dG}\left|\nabla_{\chi} Y\right|^{2} \mathrm{~d} x \mathrm{dt} \\
\leqslant \int_{0}^{T} \psi \int_{\Omega} \kappa \rho \frac{\partial \mathrm{G}(\mathbf{Y})}{\partial Y} \mathrm{~d} x \mathrm{dt}+\int_{\Omega} \rho_{0} \mathrm{G}\left(\mathbf{Y}_{0}\right) \psi(0) \mathrm{d} x
\end{gathered}
$$

for any $\psi \in \mathcal{D}[0, T), \psi \geqslant 0$, and any convex $G \in C^{2}\left(\mathbb{R}^{3}\right)$,

$$
\underline{G}=\inf _{Y \in \mathbb{R}} G^{\prime \prime}(Y) \text {. }
$$


- The chemical energy inequality

$$
\frac{1}{2} \int_{\Omega} \rho Y^{2} d x+\kappa_{\varepsilon} \int_{0}^{T} \int_{\Omega} \rho Y^{2} d x d t+d_{\varepsilon} \int_{0}^{T} \int_{\Omega}|\nabla Y|^{2} d x d t \leqslant \frac{1}{2} \int_{\Omega} \rho_{0} Y_{0}^{2} d x
$$

is satisfied in $\mathcal{D}^{\prime}(0, T)$.

\section{Main results}

In this section we introduce the main result of inviscid incompressible limit for compressible magnetohydrodynamic flows. Before we mention the main result, we introduce the generalized Helmholtz projection as follows

$$
\mathbf{v}=\mathbf{P}_{\tilde{\rho}}[\mathbf{v}]+\mathbf{P}^{\perp}[\mathbf{v}], \mathbf{P}^{\perp}[\mathbf{v}]=\nabla \Phi,
$$

where

$$
\operatorname{div}\left(\tilde{\rho} \mathbf{P}_{\tilde{\rho}}[\mathbf{v}]\right)=0, \operatorname{div}\left(\tilde{\rho} \nabla_{\chi} \Phi\right)=\operatorname{div}(\tilde{\rho} \mathbf{v}) \text { in } \Omega .
$$

Theorem 2.1. Let $\Omega=\mathbb{R}^{3}$ be the 3-dimensional whole space and $\left\{\rho_{\epsilon}, \mathbf{u}_{\epsilon}, Y_{\varepsilon}\right\}$ be a weak solution to (1.4)-(1.6) in the sense of Definition 1.1 verifying the viscosity (1.7) and the initial data:

$$
\mathbf{u}_{0, \epsilon} \rightarrow \mathbf{u}_{0} \text { in } \mathrm{L}^{2}(\Omega), \mathrm{Y}_{0, \epsilon} \rightarrow \mathrm{Y}_{0} \text { in } \mathrm{L}^{2}(\Omega), \rho_{0, \varepsilon}^{(1)} \rightarrow \rho_{0}^{(1)} \text { in } \mathrm{L}^{2}(\Omega)
$$

as $\varepsilon$ tends to 0 where

$$
\rho_{0, \varepsilon}=\tilde{\rho}+\varepsilon \rho_{0, \varepsilon}^{(1)}, \rho_{0}^{(1)} \in\left(\mathrm{L}^{2} \cap \mathrm{L}^{\infty}\right)(\Omega), \mathbf{P}_{\tilde{\rho}}\left[\mathbf{u}_{0}\right]=\mathbf{v}_{0} \in \mathrm{H}^{\mathrm{s}}\left(\Omega ; \mathbb{R}^{3}\right)
$$

for a certain $\mathrm{s}>\frac{5}{2}$. Then, one has

$$
\left\{\begin{array}{l}
\rho_{\epsilon} \rightarrow \tilde{\rho} \text { in } \mathrm{L}^{\infty}\left(0, \mathrm{~T} ;\left(\mathrm{L}^{2}+\mathrm{L}^{\gamma}\right)(\Omega)\right) \\
\sqrt{\rho_{\epsilon}} \mathbf{u}_{\epsilon} \rightarrow \sqrt{\tilde{\rho}} \mathbf{v} \text { strongly in } \mathrm{L}^{\infty}\left(0, \mathrm{~T} ; \mathrm{L}^{2}\left(\mathrm{~K} ; \mathbb{R}^{3}\right)\right), \\
\sqrt{\rho_{\epsilon}} Y_{\varepsilon} \rightarrow \sqrt{\tilde{\rho}} Y \text { strongly in } \mathrm{L}^{\infty}\left(0, \mathrm{~T} ; \mathrm{L}^{2}\left(\Omega ; \mathbb{R}^{3}\right)\right),
\end{array}\right.
$$

for any compact $\mathrm{K} \subset \mathbb{R}^{3}$ such that $\{\tilde{\rho}, \mathbf{v}, \mathrm{Y}\}$ verifies the equation (1.9) with the initial $\mathbf{P}_{\tilde{\rho}}\left(\mathbf{u}_{0}\right)$.

\section{Proof of Theorem 2.1}

In this section we are going to give a rigorous proof of Theorem 2.1. From now on, we work on any time $\mathrm{T}<\mathrm{T}_{*}$.

\subsection{Uniform bounds}

In this section, we are going to derive some estimates on the sequence $\left\{\rho_{\epsilon}, \mathbf{u}_{\epsilon}, Y_{\varepsilon}\right\}_{\epsilon>0}$. From the energy inequalities (1.12) and (1.14), we obtain

$$
\begin{aligned}
& \text { ess } \sup _{t \in(0, T)}\left\|\sqrt{\rho_{\epsilon}} \mathbf{u}_{\epsilon}(t)\right\|_{\mathrm{L}^{2}(\Omega)} \leqslant \mathrm{C}, \\
& \text { ess } \sup _{\mathrm{t} \in(0, \mathrm{~T})}\left\|\rho_{\epsilon}^{\gamma}-\tilde{\rho}^{\gamma}-\gamma \tilde{\rho}^{\gamma-1}\left(\rho_{\epsilon}-\tilde{\rho}\right)\right\|_{\mathrm{L}^{1}(\Omega)} \leqslant \epsilon^{2} \mathrm{C}, \\
& \left\|\sqrt{\mu_{\varepsilon}} \nabla \mathbf{u}_{\epsilon}\right\|_{\mathrm{L}^{2}((0, T) \times \Omega)} \leqslant \mathrm{C},\left\|\sqrt{\mu_{\varepsilon}+v_{\varepsilon}} \operatorname{div} \mathbf{u}_{\epsilon}\right\|_{\mathrm{L}^{2}((0, T) \times \Omega)} \leqslant \mathrm{C}, \\
& \left\|\sqrt{\kappa_{\varepsilon}} \sqrt{\rho_{\epsilon}} Y_{\varepsilon}\right\|_{\mathrm{L}^{\infty}\left(0, T ; \mathrm{L}^{2}(\Omega)\right)} \leqslant \mathrm{C}, \\
& \left\|\sqrt{\mathrm{d}_{\varepsilon}} \nabla Y_{\varepsilon}\right\|_{\mathrm{L}^{2}((0, T) \times \Omega)} \leqslant \mathrm{C} .
\end{aligned}
$$


Following the estimate of (3.2) and the convexity of the following function:

$$
\mathrm{r} \mapsto \mathrm{r}^{\gamma}-\tilde{\rho}^{\gamma}-\gamma \tilde{\rho}^{\gamma-1}(\mathrm{r}-\tilde{\rho}),
$$

we get

$$
\text { ess } \sup _{t \in(0, T)}\left\|\left[\frac{\rho_{\epsilon}-\tilde{\rho}}{\epsilon} 1_{\left|\rho_{\epsilon}-\tilde{\rho}\right| \leqslant 1 / 2}\right]\right\|_{L^{2}(\Omega)} \leqslant C
$$

and

$$
\text { ess } \sup _{t \in(0, T)} \int_{\Omega}\left[\frac{\left|\rho_{\epsilon}-\tilde{\rho}\right|^{\gamma}}{\epsilon^{2}} 1_{\left|\rho_{\epsilon}-\tilde{\rho}\right| \geqslant 1 / 2}\right] \mathrm{d} x \leqslant \mathrm{C} .
$$

Note that

$$
|\sqrt{x}-\sqrt{\tilde{\rho}}|^{2} \leqslant C|x-\tilde{\rho}|^{k}, k \geqslant 1
$$

and so we get

$$
\left\|\sqrt{\rho_{\epsilon}}-\sqrt{\tilde{\rho}}\right\|_{L^{\infty}\left(0, T ; \mathrm{L}^{2}(\Omega)\right)} \leqslant \varepsilon C .
$$

In accordance with (3.4) and (3.5), we obtain

$$
\rho_{\epsilon} \mathbf{u}_{\epsilon} \rightarrow \tilde{\rho} \mathbf{u} \operatorname{weakly-}(*) \text { in } \mathrm{L}^{\infty}\left(0, \mathrm{~T} ;\left(\mathrm{L}^{2}+\mathrm{L}^{2 \gamma /(\gamma+1}\right)\left(\Omega ; \mathbb{R}^{3}\right)\right),
$$

and so we deduce that

$$
\operatorname{div}(\tilde{\rho} \mathbf{u})=0
$$

in the sense of distribution.

\subsection{Dispersive estimates}

In this section we derive the dispersive estimates. To do this we first regularize the initial data. Consider the abstract operator

$$
\mathcal{A}_{\tilde{\rho}}: v \mapsto-\frac{p^{\prime}(\tilde{\rho})}{\tilde{\rho}} \operatorname{div}\left(\tilde{\rho} \nabla_{\chi} v\right),
$$

with the domain

$$
\begin{aligned}
\mathcal{D}\left(\mathcal{A}_{\tilde{\rho}}\right)= & \left\{v \in \mathrm{L}^{2}(\Omega) \mid \nabla_{\chi} v \in v \in \mathrm{L}^{2}\left(\Omega ; \mathbb{R}^{2}\right), \int_{\Omega} \tilde{\rho} \nabla_{\chi} v \cdot \nabla_{\chi}\left(\frac{p^{\prime}(\tilde{\rho})}{\tilde{\rho}} \varphi\right) \mathrm{d} x=\int_{\Omega} \mathrm{g} \varphi \mathrm{d} x\right. \\
& \text { for all } \left.\varphi \in \mathrm{C}_{\mathrm{c}}^{\infty}(\Omega) \text { and some } \mathrm{g} \in \mathrm{L}^{2}(\Omega)\right\} .
\end{aligned}
$$

Introduce a family of functions $\mathrm{G}_{\delta}$ verifying:

$$
\begin{aligned}
\mathrm{G}_{\delta} & \in \mathrm{C}_{\mathrm{c}}^{\infty}(\mathbb{R}), 0 \leqslant \mathrm{G}_{\delta} \leqslant 1, \mathrm{G}_{\delta}(-z)=\mathrm{G}_{\delta}(z), \\
\mathrm{G}_{\delta}(z) & =1 \text { for } z \in\left(-\frac{1}{\delta},-\delta\right) \cup\left(\delta, \frac{1}{\delta}\right), \\
\mathrm{G}_{\delta}(z) & =0 \text { for } z \in\left(-\infty,-\frac{2}{\delta}\right) \cup\left(-\frac{2}{\delta}, \frac{2}{\delta}\right) \cup\left(\frac{2}{\delta}, \infty\right) .
\end{aligned}
$$

We also take

$$
\psi_{\delta} \in C_{c}^{\infty}(\Omega), 0 \leqslant \psi \leqslant 1, \psi_{\delta}(x)=1 \text { for }|x|<\frac{1}{\delta}, \psi_{\delta}(x)=0 \text { for }|x|>\frac{2}{\delta} .
$$

We define the regularization $[h]_{\delta}$ by

$$
[h]_{\delta}=G_{\delta}\left(\sqrt{\mathcal{A}_{\tilde{\rho}}}\right)\left[\psi_{\delta} h\right], h>0,
$$

with $h \in \mathrm{L}^{2}(\Omega)$. Let $\left(\mathrm{q}_{\varepsilon}, \nabla_{\chi} \Phi_{\varepsilon}\right)$ solve the following acoustic equation:

$$
\varepsilon \partial_{t} \mathrm{q}_{\varepsilon}+\operatorname{div}\left[\tilde{\rho} \nabla \Phi_{\varepsilon}\right]=0,
$$




$$
\varepsilon \tilde{\rho} \partial_{\mathrm{t}} \nabla \Phi_{\varepsilon}+\tilde{\rho} \nabla\left[\frac{p^{\prime}(\tilde{\rho})}{\tilde{\rho}} \mathrm{q}_{\varepsilon}\right]=0
$$

with the initial data

$$
\mathrm{q}_{\varepsilon}(0, \cdot)=\frac{\tilde{\rho}}{p^{\prime}(\tilde{\rho})}\left[\frac{p^{\prime}(\tilde{\rho})}{\tilde{\rho}} \rho_{0, \varepsilon}^{(1)}\right]_{\delta}, \Phi_{\varepsilon}(0, \cdot)=\left[\Phi_{0, \varepsilon}\right]_{\delta}, \nabla \Phi_{0, \varepsilon}=\mathbf{P}^{\perp}\left[\mathbf{u}_{0, \varepsilon}\right]
$$

Then we have the dispersive estimates:

Theorem 3.1 ([5]). Let $\left\{\mathfrak{q}_{\varepsilon}, \nabla_{\chi} \Phi_{\varepsilon}\right\}_{\varepsilon}>0$ be the solution of system (3.9) and (3.10) with initial data in (3.11). Then, one has

$$
\sup _{t \in[0, T]}\left[\left\|\Phi_{\varepsilon}(t, \cdot)\right\|_{H^{k}(\Omega)}^{2}+\left\|q_{\varepsilon}(t, \cdot)\right\|_{H^{k}(\Omega)}^{2}\right], \leqslant c(m, \delta)\left[\left\|\nabla_{x} \Phi_{0, \varepsilon, \delta}\right\|_{L^{2}\left(\Omega ; \mathbb{R}^{2}\right)}^{2}+\left\|q_{0, \varepsilon, \delta}\right\|_{L^{2}(\Omega)}^{2}\right]
$$

and

$$
\left\|\Phi_{\mathcal{\varepsilon}}(\mathrm{t}, \cdot)\right\|_{W^{k, \infty}(\Omega)}+\left\|\mathrm{q}_{\varepsilon}(\mathrm{t}, \cdot)\right\|_{W^{k, \infty}(\Omega)} \leqslant \mathrm{h}(\varepsilon, k, \delta)\left[\left\|\nabla \Phi_{0, \varepsilon, \delta}\right\|_{\mathrm{L}^{2}\left(\Omega ; \mathbb{R}^{3}\right)}+\left\|\mathrm{q}_{0, \varepsilon, \delta}\right\|_{\mathrm{L}^{2}(\Omega)}\right],
$$

where $\mathrm{h}(\varepsilon, \mathrm{k}, \delta) \rightarrow 0$ as $\varepsilon$ tends to 0 for all fixed $\delta>0$, and $\mathrm{k}=0,1,2, \ldots$

Note that the dispersive estimates hold for the 3-dimensional whole space. From now on we delete $\delta$ to $\Phi_{\varepsilon, \delta}, \mathrm{q}_{\varepsilon, \delta}$.

\subsection{Relative entropy}

In this section we will introduce the relative entropy. Let us set

$$
H(\rho, r)=\left(P(\rho)-P^{\prime}(r)(\rho-r)-P(r)\right),
$$

where $P(s)=\frac{1}{\gamma(\gamma-1)} s^{\gamma}, p(s)=\frac{1}{\gamma} s^{\gamma}$ and

$$
\mathcal{E}_{\varepsilon}(\tau)=\int_{\Omega}\left(\frac{1}{2} \rho_{\epsilon}\left|\mathbf{u}_{\epsilon}-\mathbf{U}\right|^{2}+\frac{1}{\mathcal{\varepsilon}^{2}} H\left(\rho_{\epsilon}, r\right)+\frac{1}{2} \rho_{\epsilon}\left|Y_{\varepsilon}-Y\right|^{2}\right) d x
$$

with $\mathbf{U} \in \mathrm{C}_{\mathbf{c}}^{\infty}\left([0, \mathrm{~T}] \times \Omega ; \mathbb{R}^{3}\right)$. Using $Y$ as a test function to the equation (1.13), we get the following weak formulation of the equation (1.13)

$$
\begin{aligned}
-\int_{\Omega} \rho_{\epsilon} Y_{\varepsilon} \mathrm{Yd} x= & -\int_{\Omega} \rho_{0, \varepsilon} \mathrm{Y}_{0, \varepsilon} \mathrm{Y}_{0} \mathrm{~d} x-\int_{0}^{\tau} \int_{\Omega} \rho_{\epsilon} \mathrm{Y}_{\varepsilon} \partial_{\mathrm{t}} \mathrm{Yd} \mathrm{d} \mathrm{dt} \\
& +\mathrm{d}_{\varepsilon} \int_{0}^{\tau} \int_{\Omega} \nabla \mathrm{Y}_{\varepsilon} \cdot \nabla \mathrm{Yd} x \mathrm{dt}-\int_{0}^{\tau} \int_{\Omega} \rho_{\epsilon} \mathrm{Y}_{\varepsilon} \mathbf{u}_{\epsilon} \cdot \nabla \mathrm{Yd} x \mathrm{dt}+\kappa_{\varepsilon} \int_{0}^{\tau} \int_{\Omega} \rho_{\epsilon} \mathrm{Y}_{\varepsilon} \mathrm{Yd} \mathrm{d} \mathrm{d} \mathrm{t} .
\end{aligned}
$$

We can derive the relative entropy together with adding (1.14) and (3.14) in the sprite of Feireisl et al. [4]. For solutions $\left(\rho_{\epsilon}, \mathbf{u}_{\epsilon}\right)$ verifying (1.4)-(1.5), we have

$$
\begin{aligned}
& \mathcal{E}_{\varepsilon}(\tau)+\int_{0}^{\tau} \int_{\Omega}\left(\mu_{\varepsilon}\left|\nabla \mathbf{u}_{\epsilon}\right|^{2}+\left(\mu_{\varepsilon}+v_{\varepsilon}\right)\left|\operatorname{div} \mathbf{u}_{\epsilon}\right|^{2}\right) \mathrm{d} x \mathrm{dt} \\
& \quad+\mathrm{K}_{\varepsilon} \int_{0}^{\tau} \int_{\Omega} \rho_{\epsilon} \mathrm{Y}_{\varepsilon}^{2} \mathrm{~d} x \mathrm{~d} t+\mathrm{d}_{\varepsilon} \int_{0}^{\tau} \int_{\Omega}\left|\nabla \mathrm{Y}_{\varepsilon}\right|^{2} \mathrm{~d} x \mathrm{~d} t \leqslant \sum_{j=1}^{8} A_{j}^{\varepsilon},
\end{aligned}
$$

where

$$
\begin{aligned}
& A_{1}^{\varepsilon}=\mathcal{E}_{\varepsilon}(0), \\
& A_{2}^{\varepsilon}=\int_{0}^{\tau} \int_{\Omega} \rho_{\epsilon}\left(\partial_{t} \mathbf{U}+\mathbf{u}_{\epsilon} \cdot \nabla \mathbf{U}\right) \cdot\left(\mathbf{U}-\mathbf{u}_{\epsilon}\right) \mathrm{d} x \mathrm{dt},
\end{aligned}
$$




$$
\begin{aligned}
& A_{3}^{\varepsilon}=\int_{0}^{\tau} \int_{\Omega}\left(\mu_{\varepsilon} \nabla \mathbf{u}_{\epsilon}: \nabla \mathbf{U}+\left(\mu_{\varepsilon}+v_{\varepsilon}\right) \operatorname{div} \mathbf{u}_{\epsilon} \operatorname{div} \mathbf{U}\right) \mathrm{d} x \mathrm{dt}, \\
& A_{4}^{\varepsilon}=\frac{1}{\varepsilon^{2}} \int_{\Omega}\left(\left(r-\rho_{\epsilon}\right) \partial_{t} P^{\prime}(r)+\nabla P^{\prime}(r) \cdot\left(r \mathbf{U}-\rho_{\epsilon} \mathbf{u}_{\epsilon}\right)\right) d x, \\
& A_{5}^{\varepsilon}=-\frac{1}{\varepsilon^{2}} \int_{\Omega}\left(p\left(\rho_{\epsilon}\right)-p(r)\right) \operatorname{div} \mathbf{U d} x+\frac{1}{\varepsilon^{2}} \int_{\Omega} \rho_{\epsilon} \nabla G \cdot\left(\mathbf{u}_{\epsilon}-\mathbf{U}\right) \mathrm{d} x, \\
& A_{6}^{\varepsilon}=\int_{0}^{\tau} \int_{\Omega} \rho_{\epsilon} Y_{\varepsilon} \mathbf{v} \cdot \nabla \mathrm{Yd} x \mathrm{dt}-\int_{0}^{\tau} \int_{\Omega} \rho_{\epsilon} Y_{\varepsilon} \mathbf{u}_{\epsilon} \cdot \nabla \mathrm{Yd} x \mathrm{dt}, \\
& A_{7}^{\varepsilon}=d_{\varepsilon} \int_{0}^{\tau} \int_{\Omega} \nabla Y_{\varepsilon} \cdot \nabla Y d x d t+\kappa_{\varepsilon} \int_{0}^{\tau} \int_{\Omega} \rho_{\epsilon} Y_{\varepsilon} Y d x d t \\
& A_{8}^{\varepsilon}=\frac{1}{2} \int_{\Omega}\left(\rho_{\epsilon}-\tilde{\rho}\right) \mathrm{Y}^{2} \mathrm{~d} x-\frac{1}{2} \int_{\Omega}\left(\rho_{0, \varepsilon}-\tilde{\rho}\right) \mathrm{Y}^{2} \mathrm{~d} x,
\end{aligned}
$$

where $r$ satisfies

$$
r>0 \text { in }[0, T] \times \Omega, r-\tilde{\rho} \in C_{c}^{\infty}([0, T] \times \Omega) .
$$

\subsection{Computation of relative entropy}

In the relative entropy inequality (3.15), we take

$$
\mathrm{r}=\tilde{\rho}+\varepsilon \mathbf{q}_{\varepsilon}, \mathbf{U}=\mathbf{v}+\nabla \Phi_{\varepsilon} .
$$

Following the estimates of (2.1), the initial terms of $A_{1}^{\varepsilon}$ can be handled as follows

$$
\begin{aligned}
\left\|\sqrt{\rho_{0, \varepsilon}}\left(\mathbf{u}_{0, \varepsilon}-\mathbf{v}_{0}-\nabla \Phi_{0, \varepsilon}\right)\right\|_{\mathrm{L}^{2}(\Omega)} & \leqslant \mathrm{C}\left\|\mathbf{u}_{0, \varepsilon}-\mathbf{u}_{0}\right\|_{\mathrm{L}^{2}(\Omega)} \\
+\mathrm{C}\left\|\mathbf{P}^{\perp}\left[\mathbf{u}_{0}\right]-\mathbf{P}^{\perp}\left[\mathbf{u}_{0, \varepsilon}\right]\right\|_{\mathrm{L}^{2}(\Omega)}+\mathrm{C}\left\|\mathbf{P}^{\perp}\left[\mathbf{u}_{0, \varepsilon}\right]-\left[\mathbf{P}^{\perp}\left[\mathbf{u}_{0, \varepsilon}\right]\right]_{\delta}\right\|_{\mathrm{L}^{2}(\Omega)} & =\chi(\varepsilon, \delta),
\end{aligned}
$$

with

$$
\lim _{\varepsilon \rightarrow 0, \delta \rightarrow 0} \chi(\varepsilon, \delta)=0
$$

where we have used (2.2),

$$
\int_{\Omega} \frac{1}{\varepsilon^{2}} H\left(\rho_{0, \varepsilon}, r_{0, \varepsilon}\right) \mathrm{d} x \leqslant C \int_{\Omega}\left(\left|\rho_{0, \varepsilon}^{(1)}-\rho^{(1)}\right|^{2}+\left|\frac{\tilde{\rho}}{p^{\prime}(\tilde{\rho})}\left[\frac{p^{\prime}(\tilde{\rho})}{\tilde{\rho}} \rho_{0, \varepsilon}^{(1)}\right]_{\delta}-\rho^{(1)}\right|^{2}\right) \mathrm{d} x=\chi(\varepsilon, \delta),
$$

and

$$
\int_{\Omega} \rho_{0, \varepsilon}\left|Y_{0, \varepsilon}-Y_{0}\right|^{2} \mathrm{~d} x \leqslant C \int_{\Omega}\left|\rho_{0, \varepsilon}-\tilde{\rho} \| Y_{0, \varepsilon}-Y_{0}\right|^{2} \mathrm{~d} x+C \int_{\Omega}\left|\mathrm{Y}_{0, \varepsilon}-\mathrm{Y}_{0}\right|^{2} \mathrm{~d} x=\chi(\varepsilon, \delta) .
$$

Thus, we get $A_{1}^{\varepsilon}=\chi(\varepsilon, \delta)$. We next control the velocity terms $A_{2}^{\varepsilon}$

$$
\begin{aligned}
A_{2}^{\varepsilon} & =\int_{0}^{\tau} \int_{\Omega} \rho_{\epsilon}\left(\partial_{t} \mathbf{U}+\mathbf{u}_{\epsilon} \cdot \nabla \mathbf{U}\right)\left(\mathbf{U}-\mathbf{u}_{\epsilon}\right) \mathrm{d} x \mathrm{dt} \\
& =\int_{0}^{\tau} \int_{\Omega} \rho_{\epsilon}\left(\mathbf{U}-\mathbf{u}_{\epsilon}\right) \otimes\left(\mathbf{U}-\mathbf{u}_{\epsilon}\right): \nabla \mathbf{U} \mathrm{d} x \mathrm{dt}+\int_{0}^{\tau} \int_{\Omega}\left(\rho_{\epsilon}\left(\mathbf{U}-\mathbf{u}_{\epsilon}\right) \cdot \partial_{\mathrm{t}} \mathbf{U}+\rho_{\epsilon}\left(\mathbf{U}-\mathbf{u}_{\epsilon}\right): \nabla \mathbf{U}\right) \mathrm{d} x \mathrm{dt} \\
& \leqslant \mathrm{C} \int_{0}^{\tau} \mathcal{E}_{\mathcal{\varepsilon}}(\mathrm{t}) \mathrm{dt}+\sum_{\mathrm{k}=1}^{5} J_{k^{\prime}}^{\varepsilon}
\end{aligned}
$$

where

$$
\begin{array}{ll}
\mathrm{J}_{1}^{\varepsilon}=\int_{0}^{\tau} \int_{\Omega} \rho_{\epsilon}\left(\mathbf{U}-\mathbf{u}_{\epsilon}\right) \cdot\left(\partial_{\mathrm{t}} \mathbf{v}+\mathbf{v} \cdot \nabla \mathbf{v}\right) \mathrm{d} x \mathrm{dt}, & \mathrm{J}_{2}^{\varepsilon}=\int_{0}^{\tau} \int_{\Omega} \rho_{\epsilon}\left(\mathbf{U}-\mathbf{u}_{\epsilon}\right) \cdot \partial_{\mathrm{t}} \nabla \Phi_{\varepsilon} \mathrm{d} x \mathrm{dt}, \\
\mathrm{J}_{3}^{\varepsilon}=\int_{0}^{\tau} \int_{\Omega} \rho_{\epsilon}\left(\mathbf{U}-\mathbf{u}_{\epsilon}\right) \otimes \nabla \Phi_{\varepsilon}: \nabla \mathbf{v} \mathrm{d} x \mathrm{dt}, & \mathrm{J}_{4}^{\varepsilon}=\int_{0}^{\tau} \int_{\Omega} \rho_{\epsilon}\left(\mathbf{U}-\mathbf{u}_{\epsilon}\right) \otimes \mathbf{v}: \nabla^{2} \Phi_{\varepsilon} \mathrm{d} x \mathrm{dt},
\end{array}
$$




$$
\mathrm{J}_{5}^{\varepsilon}=\frac{1}{2} \int_{0}^{\tau} \int_{\Omega} \rho_{\epsilon}\left(\mathbf{U}-\mathbf{u}_{\epsilon}\right) \cdot \nabla\left|\nabla \Phi_{\varepsilon}\right|^{2} \mathrm{~d} x \mathrm{dt}
$$

For $J_{1}^{\varepsilon}$, employing (3.4), (3.5), (3.12), and (3.13) gives

$$
\begin{aligned}
\int_{0}^{\tau} \int_{\Omega} \rho_{\epsilon} \nabla \Phi_{\varepsilon} \cdot\left(\partial_{\mathrm{t}} \mathbf{v}+\mathbf{v} \cdot \nabla \mathbf{v}\right) \mathrm{d} x \mathrm{~d} t & =-\epsilon \int_{0}^{\tau} \int_{\Omega} \frac{\rho_{\epsilon}-\tilde{\rho}}{\varepsilon} \nabla \Phi_{\varepsilon} \cdot \nabla \Pi \mathrm{d} x \mathrm{~d} t-\int_{0}^{\tau} \int_{\Omega} \tilde{\rho} \nabla \Phi_{\varepsilon} \cdot \nabla \Pi \mathrm{d} x \mathrm{~d} \mathrm{t} \\
& =\int_{0}^{\tau} \int_{\Omega}^{\tau} \operatorname{div}\left(\tilde{\rho} \nabla \Phi_{\varepsilon}\right) \Pi \mathrm{d} x \mathrm{dt}+\chi(\varepsilon, \delta) \\
& =-\varepsilon \int_{0}^{\tau} \int_{\Omega} \partial_{\mathrm{t}} \mathrm{q}_{\varepsilon} \Pi \mathrm{d} x \mathrm{~d} \mathrm{t}+\chi(\varepsilon, \delta) \\
& =-\varepsilon\left[\int_{\Omega} \mathrm{q}_{\varepsilon} \Pi\right]_{0}^{\tau}+\varepsilon \int_{0}^{\tau} \int_{\Omega} \mathrm{q}_{\varepsilon} \partial_{\mathrm{t}} \Pi \mathrm{d} x \mathrm{dt}=\chi(\varepsilon, \delta) .
\end{aligned}
$$

Furthermore, using(3.4), (3.5), and (3.8), it follows that

$$
\begin{aligned}
\int_{0}^{\tau} \int_{\Omega} \rho_{\epsilon}\left(\mathbf{v}-\mathbf{u}_{\epsilon}\right) \cdot\left(\partial_{\mathrm{t}} \mathbf{v}+\mathbf{v} \cdot \nabla \mathbf{v}\right) \mathrm{d} x \mathrm{~d} \mathrm{t},= & -\varepsilon \int_{0}^{\tau} \int_{\Omega} \frac{\rho_{\epsilon}-\tilde{\rho}}{\varepsilon} \mathbf{v} \cdot \nabla \Pi \mathrm{d} x \mathrm{~d} t-\int_{0}^{\tau} \int_{\Omega} \tilde{\rho} \mathbf{v} \cdot \nabla \Pi \mathrm{d} x \mathrm{~d} t \\
& +\int_{0}^{\tau} \int_{\Omega} \rho_{\epsilon} \mathbf{u}_{\epsilon} \cdot \nabla \Pi \mathrm{d} x \mathrm{~d} t+\chi(\varepsilon, \delta)=\chi(\varepsilon, \delta) .
\end{aligned}
$$

Next let us control $J_{2}^{\varepsilon}$ for the velocity term. Indeed,

$$
\begin{aligned}
\int_{0}^{\tau} \int_{\Omega} \rho_{\epsilon}\left(\mathbf{U}-\mathbf{u}_{\epsilon}\right) \cdot \partial_{\mathrm{t}} \nabla \Phi_{\varepsilon} \mathrm{d} x \mathrm{dt}= & -\int_{0}^{\tau} \int_{\Omega} \rho_{\epsilon} \mathbf{u}_{\epsilon} \cdot \partial_{\mathrm{t}} \nabla \Phi_{\varepsilon} \mathrm{d} x \mathrm{dt}+\int_{0}^{\tau} \int_{\Omega} \rho_{\epsilon} \mathbf{v} \cdot \partial_{\mathrm{t}} \nabla \Phi_{\varepsilon} \mathrm{d} x \mathrm{dt} \\
& +\frac{1}{2} \int_{0}^{\tau} \int_{\Omega} \rho_{\epsilon} \partial_{\mathrm{t}}\left|\nabla \Phi_{\varepsilon}\right|^{2} \mathrm{~d} x \mathrm{dt} .
\end{aligned}
$$

The second integral on the right hand side of (3.16) can be handled by

$$
\begin{aligned}
\int_{0}^{\tau} \int_{\Omega} \rho_{\epsilon} \mathbf{v} \cdot \partial_{t} \nabla \Phi_{\varepsilon} \mathrm{d} x \mathrm{dt} & =\int_{0}^{\tau} \int_{\Omega}\left(\rho_{\epsilon}-\tilde{\rho}\right) \mathbf{v} \cdot \partial_{\mathrm{t}} \nabla \Phi_{\varepsilon} \mathrm{d} x \mathrm{dt} \\
& =-\int_{0}^{\tau} \int_{\Omega} \frac{\left(\rho_{\epsilon}-\tilde{\rho}\right)}{\varepsilon} \mathbf{v} \cdot \nabla\left[\frac{p^{\prime}(\tilde{\rho})}{\tilde{\rho}} \mathbf{q}_{\varepsilon}\right] \mathrm{d} x \mathrm{dt}=\chi(\varepsilon, \delta),
\end{aligned}
$$

where we have here used (3.4), (3.5), and the dispersive regularity (3.13). Next, we estimates the third integral on the right hand side of (3.16). Using (3.4), (3.5), and the dispersive regularity (3.13) again provides

$$
\begin{aligned}
\frac{1}{2} \int_{0}^{\tau} \int_{\Omega} \rho_{\epsilon} \partial_{\mathrm{t}}\left|\nabla \Phi_{\varepsilon}\right|^{2} \mathrm{~d} x \mathrm{dt} & =\frac{\varepsilon}{2} \int_{0}^{\tau} \int_{\Omega} \frac{\rho_{\epsilon}-\tilde{\rho}}{\varepsilon} \partial_{\mathrm{t}}\left|\nabla \Phi_{\varepsilon}\right|^{2} \mathrm{~d} x \mathrm{dt}+\frac{1}{2} \int_{0}^{\tau} \int_{\Omega} \tilde{\rho} \partial_{\mathrm{t}}\left|\nabla \Phi_{\varepsilon}\right|^{2} \mathrm{~d} x \mathrm{dt} \\
& =-\int_{0}^{\tau} \int_{\Omega} \frac{\rho_{\epsilon}-\tilde{\rho}}{\varepsilon} \nabla \Phi_{\varepsilon} \cdot \nabla\left[\frac{p^{\prime}(\tilde{\rho})}{\tilde{\rho}} \mathrm{q}_{\varepsilon}\right] \mathrm{d} x \mathrm{dt}+\frac{1}{2} \int_{0}^{\tau} \int_{\Omega} \tilde{\rho} \partial_{\mathrm{t}}\left|\nabla \Phi_{\varepsilon}\right|^{2} \mathrm{~d} x \mathrm{dt} \\
& =\frac{1}{2} \int_{0}^{\tau} \int_{\Omega} \tilde{\rho} \partial_{\mathrm{t}}\left|\nabla \Phi_{\varepsilon}\right|^{2} \mathrm{~d} x \mathrm{dt}+\chi(\varepsilon, \delta) .
\end{aligned}
$$

Using the regularity (1.10), the dispersive regularity (3.13), and (3.1), the term of $J_{3}^{\varepsilon}$ can be estimated as

$$
J_{3}^{\varepsilon}=\int_{0}^{\tau} \int_{\Omega}\left(\rho_{\epsilon}-\tilde{\rho}\right) \mathbf{U} \otimes \nabla \Phi_{\varepsilon}: \nabla \mathbf{v} \mathrm{d} x \mathrm{~d} t+\int_{0}^{\tau} \int_{\Omega} \tilde{\rho} \mathbf{U} \otimes \nabla \Phi_{\varepsilon}: \nabla \mathbf{v d} x \mathrm{dt}=\chi(\varepsilon, \delta) .
$$

Similarly, we get

$$
\mathrm{J}_{4}^{\varepsilon}+\mathrm{J}_{5}^{\varepsilon}=\chi(\varepsilon, \delta)
$$


and the term of $A_{2}^{\varepsilon}$ is estimated by

$$
A_{2}^{\varepsilon}=\left[\frac{1}{2} \int_{\Omega} \tilde{\rho}\left|\nabla \Phi_{\varepsilon}\right|^{2} \mathrm{~d} x\right]_{\mathrm{t}=0}^{\tau}-\int_{0}^{\tau} \int_{\Omega} \rho_{\epsilon} \mathbf{u}_{\epsilon} \cdot \partial_{\mathrm{t}} \nabla \Phi_{\varepsilon} \mathrm{d} x \mathrm{dt}+\chi(\varepsilon, \delta) .
$$

Let us show that the viscosity term $A_{3}^{\varepsilon}$ vanishes,

$$
\begin{aligned}
& \left|\int_{\Omega}\left(\mu_{\varepsilon} \nabla \mathbf{u}_{\epsilon}: \nabla \mathbf{U}+\left(\mu_{\varepsilon}+\lambda_{\varepsilon}\right) \operatorname{div} \mathbf{u}_{\epsilon} \operatorname{div} \mathbf{U}\right) \mathrm{d} x\right| \\
& \quad \leqslant \frac{1}{2} \mu_{\varepsilon} \int_{\Omega}\left|\nabla \mathbf{u}_{\epsilon}\right|^{2} \mathrm{~d} x+\frac{1}{2}\left(\mu_{\varepsilon}+\lambda_{\varepsilon}\right) \int_{\Omega}\left|\operatorname{div} \mathbf{u}_{\epsilon}\right|^{2} \mathrm{~d} x+\mathrm{C}\left(\mu_{\varepsilon}+\lambda_{\varepsilon}\right) \int_{\Omega}|\nabla \mathbf{U}|^{2} \mathrm{~d} x,
\end{aligned}
$$

where we have here used (1.7), (1.10), (3.3), (3.12), and the fact (3.6), together with (3.4) and (3.5). Indeed, the first and second terms can be moved to the left hand side in (3.17) and the third term vanishes. In (3.17), we know that, from (1.10) and (3.12), $\nabla \mathbf{U} \in \mathrm{H}^{2}(\Omega)$ together with the Sobolev embedding, which implies that

$$
\|\nabla \mathbf{U}\|_{L^{\infty}(\Omega)} \leqslant \mathrm{C} .
$$

For the term of $A_{4}^{\varepsilon}, A_{5}^{\varepsilon}$, we are going to use the same result in [5] such that

$$
\begin{aligned}
A_{4}^{\varepsilon}+A_{5}^{\varepsilon}= & \frac{1}{\varepsilon^{2}} \int_{0}^{\tau} \int_{\Omega}\left(\left(r-\rho_{\epsilon}\right) \partial_{t} P^{\prime}(r)+\nabla P^{\prime}(r) \cdot\left(r \mathbf{U}-\rho_{\epsilon} \mathbf{u}_{\epsilon}\right)\right) d x d t \\
& -\frac{1}{\varepsilon^{2}} \int_{0}^{\tau} \int_{\Omega}\left(p\left(\rho_{\epsilon}\right)-p(r)\right) \operatorname{div} \mathbf{U d} x d t+\frac{1}{\varepsilon^{2}} \int_{\Omega} \rho_{\epsilon} \nabla \mathrm{G} \cdot\left(\mathbf{u}_{\epsilon}-\mathbf{U}\right) \mathrm{d} x \\
= & -\left[\frac{1}{2} \int_{\Omega} \tilde{\rho}\left|\nabla \Phi_{\varepsilon}\right|^{2} \mathrm{~d} x\right]_{\mathrm{t}=0}^{\tau}+\int_{0}^{\tau} \int_{\Omega} \rho_{\epsilon} \mathbf{u}_{\epsilon} \cdot \partial_{\mathrm{t}} \nabla \Phi_{\varepsilon} \mathrm{d} x \mathrm{dt}+\chi(\varepsilon, \delta) .
\end{aligned}
$$

We now handle the convergence of the reactant mass fraction function $A_{6}^{\varepsilon}$. The term of $A_{6}^{\varepsilon}$ can be written by $A_{6}^{\varepsilon}=\sum_{j=1}^{4} B_{j}^{\varepsilon}$, where

$$
\begin{aligned}
& \mathrm{B}_{1}^{\varepsilon}=\int_{0}^{\tau} \int_{\Omega} \sqrt{\rho_{\epsilon}}\left(\mathrm{Y}_{\varepsilon}-\mathrm{Y}\right) \sqrt{\rho_{\epsilon}}\left(\mathbf{U}-\mathbf{u}_{\epsilon}\right) \cdot \nabla \mathrm{Yd} x \mathrm{dt}, \\
& \mathrm{B}_{2}^{\varepsilon}=-\int_{0}^{\tau} \int_{\Omega} \sqrt{\rho_{\epsilon}}\left(\mathrm{Y}_{\varepsilon}-\mathrm{Y}\right) \sqrt{\rho_{\epsilon}} \nabla \Phi_{\varepsilon} \cdot \nabla \mathrm{Yd} x \mathrm{dt}, \\
& \mathrm{B}_{3}^{\varepsilon}=-\int_{0}^{\tau} \int_{\Omega}^{\tau} \rho_{\epsilon} \mathrm{Yv} \cdot \nabla \mathrm{Yd} \mathrm{d} \mathrm{dt}, \\
& \mathrm{B}_{4}^{\varepsilon}=-\int_{0}^{\tau} \int_{\Omega} \rho_{\epsilon} \mathrm{Yu}_{\epsilon} \cdot \nabla \mathrm{Yd} \mathrm{d} \mathrm{dt} .
\end{aligned}
$$

Applying the Young's inequality and using the regularity in (1.10), the first intergal can be bounded by the modulated energy

$$
B_{1}^{\varepsilon} \leqslant C \int_{0}^{\tau} \int_{\Omega} \rho_{\epsilon}\left|Y_{\varepsilon}-Y\right|^{2} d x d t+C \int_{0}^{\tau} \int_{\Omega} \rho_{\epsilon}\left|\mathbf{u}_{\epsilon}-\mathbf{U}\right|^{2} \mathrm{~d} x \mathrm{dt} \leqslant C \int_{0}^{\tau} \mathcal{E}_{\varepsilon}(t) d t .
$$

We use the Young's inequality again to obtain that:

$$
\begin{aligned}
& \mathrm{B}_{2}^{\varepsilon} \leqslant \mathrm{C} \int_{0}^{\tau} \int_{\Omega} \rho_{\epsilon}\left|\mathrm{Y}_{\mathcal{\varepsilon}}-\mathrm{Y}\right|^{2} \mathrm{~d} x \mathrm{dt}+\mathrm{C} \int_{0}^{\tau} \int_{\Omega} \rho_{\epsilon}\left|\nabla \Phi_{\varepsilon}\right|^{2}|\nabla \mathrm{Y}|^{2} \mathrm{~d} x \mathrm{dt}, \\
& \leqslant C \int_{0}^{\tau} \varepsilon_{\varepsilon}(t) d t+C\left\|\left(\rho_{\epsilon}-\tilde{\rho}\right) 1_{\left|\rho_{\epsilon}-\tilde{\rho}\right| \leqslant 1 / 2}\right\|_{\mathrm{L}^{2}\left(0, \mathrm{~T} ; \mathrm{L}^{2}(\Omega)\right)}\|\nabla \mathrm{Y}\|_{\mathrm{L}^{2}\left(0, \mathrm{~T} ; \mathrm{L}^{2}(\Omega)\right)} \\
& +C\left\|\left(\rho_{\epsilon}-\tilde{\rho}\right) 1_{\left|\rho_{\epsilon}-\tilde{\rho}\right| \geqslant 1 / 2}\right\|_{\mathrm{L}^{\gamma}\left(0, \mathrm{~T} ; \mathrm{L}^{\gamma}(\Omega)\right)}\|\nabla \mathrm{Y}\|_{\mathrm{L}^{\frac{2 \gamma}{\gamma-1}\left(0, \mathrm{~T} ; \mathrm{L} \mathrm{L}^{\gamma-1}(\Omega)\right)}}+\mathrm{C} \int_{0}^{\tau} \int_{\Omega}\left|\nabla \Phi_{\varepsilon}\right|^{2}|\nabla \mathrm{Y}|^{2} \mathrm{~d} x \mathrm{dt}, \\
& \leqslant C \int_{0}^{\tau} \varepsilon_{\varepsilon}(t) d t+\chi(\varepsilon, \delta)
\end{aligned}
$$


where we have used the regularity in (1.10) and the dispersive regularity (3.13). Using again divv $=0$ and (3.7) together with the similar method of $B_{3}^{\varepsilon}, B_{4}^{\varepsilon}$, we get

$$
\mathrm{B}_{3}^{\varepsilon}=\chi(\varepsilon, \delta)-\int_{0}^{\tau} \int_{\Omega} \tilde{\rho} \mathbf{v} \cdot \nabla \frac{Y^{2}}{2} \mathrm{~d} x \mathrm{dt}=\chi(\varepsilon, \delta)
$$

and

$$
\mathrm{B}_{4}^{\varepsilon}=-\int_{0}^{\tau} \int_{\Omega} \rho_{\epsilon} \mathbf{u}_{\epsilon} \cdot \nabla \frac{\mathrm{Y}^{2}}{2} \mathrm{~d} x \mathrm{dt}=\chi(\varepsilon, \delta),
$$

where we have used (3.8). Consequently, we have

$$
A_{6}^{\varepsilon} \leqslant C \int_{0}^{\tau} \varepsilon_{\varepsilon}(t) d t+\chi(\varepsilon, \delta) .
$$

For the term of $A_{7}^{\varepsilon}$, using the estimate of (3.8) yields that:

$$
\begin{aligned}
A_{7}^{\varepsilon} \leqslant & \frac{d_{\varepsilon}}{2} \int_{0}^{\tau} \int_{\Omega}\left|\nabla Y_{\varepsilon}\right|^{2} \mathrm{~d} x \mathrm{~d} t+\frac{K_{\varepsilon}}{2} \int_{0}^{\tau} \int_{\Omega} \rho_{\epsilon} Y_{\varepsilon}^{2} \mathrm{~d} x \mathrm{dt} \\
& +\left\|\sqrt{\rho_{\epsilon}}-\sqrt{\tilde{\rho}}\right\|_{\mathrm{L}^{\infty}\left(0, \mathrm{~T} ; \mathrm{L}^{2}(\Omega)\right)}\left\|\sqrt{K_{\varepsilon}} \sqrt{\rho_{\epsilon}} Y_{\varepsilon}\right\|_{\mathrm{L}^{\infty}\left(0, \mathrm{~T} ; \mathrm{L}^{2}(\Omega)\right)}+\chi(\varepsilon, \delta) \\
\leqslant & \frac{\mathrm{d}_{\varepsilon}}{2} \int_{0}^{\tau} \int_{\Omega}\left|\nabla \mathrm{Y}_{\varepsilon}\right|^{2} \mathrm{~d} x \mathrm{dt}+\frac{K_{\varepsilon}}{2} \int_{0}^{\tau} \int_{\Omega} \rho_{\epsilon} Y_{\varepsilon}^{2} \mathrm{~d} x \mathrm{dt}+\chi(\varepsilon, \delta) .
\end{aligned}
$$

Finally, by (1.10), (3.4), and (3.5), it remains to bound the term of $A_{8}^{\varepsilon}$

$$
\begin{aligned}
\left|\int_{\Omega}\left(\rho_{\epsilon}-\tilde{\rho}\right) \mathrm{Y}^{2} \mathrm{~d} x\right| \leqslant & \left\|\left(\rho_{\epsilon}-\tilde{\rho}\right) 1_{\left|\rho_{\epsilon}-\tilde{\rho}\right| \leqslant 1 / 2}\right\|_{\mathrm{L}^{2}\left(0, \mathrm{~T} ; \mathrm{L}^{2}(\Omega)\right)}\|\mathrm{Y}\|_{\mathrm{L}^{2}\left(0, \mathrm{~T} ; \mathrm{L}^{2}(\Omega)\right)} \\
& +\mathrm{C}\left\|\left(\rho_{\epsilon}-\tilde{\rho}\right) 1_{\left|\rho_{\epsilon}-\tilde{\rho}\right| \geqslant 1 / 2}\right\|_{\mathrm{L}^{\gamma}\left(0, \mathrm{~T} ; \mathrm{L}^{\gamma}(\Omega)\right)}\|\mathrm{Y}\|_{\left.\mathrm{L}^{\frac{2 \gamma}{\gamma-1}(0, \mathrm{~T} ; \mathrm{L} \gamma-1}(\Omega)\right)}=\chi(\varepsilon, \delta)
\end{aligned}
$$

and similarly by the initial condition (2.1),

$$
\int_{\Omega}\left(\rho_{0, \varepsilon}-\tilde{\rho}\right) \mathrm{Y}_{0}^{2} \mathrm{~d} x=\chi(\varepsilon, \delta) .
$$

Thus we have

$$
A_{8}^{\varepsilon}=\chi(\varepsilon, \delta) .
$$

Then, summarizing the estimates for $\Sigma_{j=1}^{3} A_{j}^{\varepsilon}$, we show that the relative entropy (3.15) provides

$$
\begin{aligned}
& \mathcal{E}_{\varepsilon}(\tau)+\frac{1}{2} \int_{0}^{\tau} \int_{\Omega}\left(\mu_{\varepsilon}\left|\nabla \mathbf{u}_{\epsilon}\right|^{2}+\left(\mu_{\varepsilon}+v_{\varepsilon}\right)\left|\operatorname{div} \mathbf{u}_{\epsilon}\right|^{2}\right) \mathrm{d} x \mathrm{dt} \\
& \quad+\frac{1}{2} \kappa_{\varepsilon} \int_{0}^{\tau} \int_{\Omega} \rho_{\epsilon} Y_{\varepsilon}^{2} \mathrm{~d} x \mathrm{dt}+\frac{1}{2} \mathrm{~d}_{\varepsilon} \int_{0}^{\tau} \int_{\Omega}\left|\nabla \mathrm{Y}_{\varepsilon}\right|^{2} \mathrm{~d} x \mathrm{~d} t \leqslant C \int_{0}^{\tau} \mathcal{E}_{\varepsilon}(\mathrm{t}) \mathrm{d} x+\chi(\varepsilon, \delta) .
\end{aligned}
$$

\subsection{Convergence of (2.3)}

Let us apply the Gronwall's inequality to (3.18) in order to obtain:

$$
\mathcal{E}_{\varepsilon}(\tau) \leqslant \chi(\varepsilon, \delta) \exp (\tau C)
$$

for any $\tau \in(0, T)$. We are now able to prove the local convergence of $\sqrt{\rho_{\epsilon}} \mathbf{u}_{\epsilon}$. Note that

$$
\begin{aligned}
\int_{K}\left|\sqrt{\rho_{\epsilon}} \mathbf{u}_{\epsilon}-\sqrt{\tilde{\rho}} \mathbf{v}\right|^{2} \mathrm{~d} x & \leqslant \int_{K}\left|\sqrt{\rho_{\epsilon}} \mathbf{u}_{\epsilon}-\sqrt{\rho_{\epsilon}} \mathbf{v}-\sqrt{\rho_{\epsilon}} \nabla \Phi_{\varepsilon}-\left(\sqrt{\tilde{\rho}}-\sqrt{\rho_{\epsilon}}\right)\left(\mathbf{v}+\nabla \Phi_{\varepsilon}\right)+\sqrt{\tilde{\rho}} \nabla \Phi_{\varepsilon}\right|^{2} \mathrm{~d} x \\
& \leqslant \int_{\Omega} \rho_{\epsilon}\left|\mathbf{u}_{\epsilon}-\mathbf{U}\right|^{2} \mathrm{~d} x+\mathrm{C}(\mathrm{K})\left\|\Phi_{\varepsilon}\right\|_{W^{1, \infty}(\Omega)}+\chi(\varepsilon, \delta) \\
& \leqslant \int_{\Omega} \rho_{\epsilon}\left|\mathbf{u}_{\epsilon}-\mathbf{U}\right|^{2} \mathrm{~d} x+\chi(\varepsilon, \delta)
\end{aligned}
$$


and any compact subset $\mathrm{K} \subset \Omega$, any $\mathrm{T}<\mathrm{T}_{*}$, and

$$
\int_{\Omega}\left|\sqrt{\rho_{\epsilon}} Y_{\varepsilon}-\sqrt{\tilde{\rho}} Y\right|^{2} \mathrm{~d} x \leqslant \int_{\Omega} \rho_{\epsilon}\left|Y_{\varepsilon}-Y\right|^{2} \mathrm{~d} x+\chi(\varepsilon, \delta) \leqslant C \mathcal{E}_{\varepsilon}(\tau)+\chi(\varepsilon, \delta) \leqslant \chi(\varepsilon, \delta),
$$

where we have here used (3.4), (3.5), (3.12), and (3.6). Consequently, using (3.19) and (3.20) together with (3.13) and passing to the limit for $\varepsilon \rightarrow 0, \delta \rightarrow 0$, we get

$$
\limsup _{\varepsilon \rightarrow 0}\left\|\sqrt{\rho_{\epsilon}} \mathbf{u}_{\epsilon}-\sqrt{\tilde{\rho}} \mathbf{v}\right\|_{\mathrm{L}^{\infty}\left(0, \mathrm{~T} ; \mathrm{L}_{\mathrm{loc}}^{2}(\Omega)\right)}=0 \quad \text { and } \quad \limsup _{\varepsilon \rightarrow 0}\left\|\sqrt{\rho_{\epsilon}} Y_{\varepsilon}-\sqrt{\tilde{\rho}} Y\right\|_{\mathrm{L}^{\infty}\left(0, \mathrm{~T} ; \mathrm{L}^{2}(\Omega)\right)}=0 .
$$

Consequently, we prove the second and third parts of (2.3).

\section{Acknowledgment}

The work of Young-Sam Kwon was supported by the research fund of Dong-A University.

\section{References}

[1] D. Donatelli, K. Trivisa, On the motion of a viscous compressible radiative-reacting gas, Comm. Math. Phys., 265 (2006), 463-491. 1

[2] D. Donatelli, K. Trivisa, A multidimensional model for the combustion of compressible fluids, Arch. Ration. Mech. Anal., 185 (2007), 379-408. 1

[3] D. Donatelli, K. Trivisa, From the dynamics of gaseous stars to the incompressible Euler equations, J. Differential Equations, 245 (2008), 1356-1385. 1, 1

[4] E. Feireisl, B. J. Jin, A. Novotný, Relative entropies, suitable weak solutions, and weak-strong uniqueness for the compressible Navier-Stokes system, J. Math. Fluid Mech., 14 (2012), 717-730. 3.3

[5] E. Feireisl, B. J. Jin, A. Novotný, Inviscid incompressible limits of strongly stratified fluids, Asymptot. Anal., 89 (2014), 307-329. 1, 3.1, 3.4

[6] E. Feireisl, A. Novotný, Inviscid incompressible limits of the full Navier-Stokes-Fourier system, Comm. Math. Phys., 321 (2013), 605-628. 1

[7] E. Feireisl, H. Petzeltová, Low Mach number asymptotics for reacting compressible fluid flows, Discrete Contin. Dyn. Syst., 26 (2010), 455-480. 1

[8] S. Jiang, Q.-C. Ju, F.-C. Li, Incompressible limit of the compressible magnetohydrodynamic equations with periodic boundary conditions, Comm. Math. Phys., 297 (2010), 371-400. 1

[9] S. Jiang, Q.-C. Ju, F.-C. Li, Incompressible limit of the compressible magnetohydrodynamic equations with vanishing viscosity coefficients, SIAM J. Math. Anal., 42 (2010), 2539-2553.

[10] S. Jiang, Q.-C. Ju, F.-C. Li, Z.-P. Xin, Low Mach number limit for the full compressible magnetohydrodynamic equations with general initial data, Adv. Math., 259 (2014), 384-420. 1

[11] N. Masmoudi, Incompressible, inviscid limit of the compressible Navier-Stokes system, Ann. Inst. H. Poincaré Anal. Non Linéaire, 18 (2001), 199-224. 1 